\title{
Diclofenac in adolescents: diagnosing and treating gastrointestinal adverse drug reactions can prevent future deaths
}

High doses or prolonged use of diclofenac, a commonly prescribed non-steroidal antiinflammatory drug (NSAID), may result in gastrointestinal complications, which, if ignored, can be fatal. A preventable death attributed to diclofenac in a 16-year-old with complex needs has been identified, and is described in this article. Adverse drug reactions from NSAIDs should be considered and treated in children and adolescents, especially those with complex needs.

\section{Elizabeth T. Thomas ${ }^{\star 1}$, Georgia C. Richards ${ }^{1}$}

${ }^{1}$ Centre for Evidence-Based Medicine, Nuffield Department of Primary Care Health Sciences, University of Oxford, Radcliffe Observatory Quarter, Woodstock Road, Oxford, OX2 6HT, UK.

*corresponding author: elizabeth.thomas@phc.ox.ac.uk

Word count: 1,004

This article is part of the Coroners' Concerns to Prevent Harms series. ${ }^{1}$ It discusses a Prevention of Future Death (PFD) report related to harm from diclofenac in an adolescent. ${ }^{2}$

Diclofenac is a non-steroidal anti-inflammatory drug (NSAID) that is commonly prescribed for its analgesic, antipyretic, and anti-inflammatory effects. About 658,000 prescriptions were dispensed for diclofenac in English primary care in the 12 months from September 2019 to August 2020, costing £8.3 million. ${ }^{3}$ Diclofenac is thought to exert its action by reducing prostaglandin synthesis through inhibition of the cyclo-oxygenase enzymes (COX-1 and COX2). It is classified as a non-selective COX inhibitor, though in-vitro studies have shown it preferentially inhibits COX-2 more than COX-1.4

Diclofenac is most commonly prescribed for pain relief but the specific indications for its use in children and adolescents vary by age, as recommended in the British National Formulary (BNF) for Children, see Box $1 .{ }^{5}$ Diclofenac, along with other NSAIDs, is associated with increased risks of bleeding, cardiovascular events, renal impairment, and gastrointestinal complications, including peptic ulcer disease. ${ }^{6}$ These harms are well reported in the elderly, and NSAIDs have been implicated in $30 \%$ of all hospital admissions due to preventable adverse drug reactions 
(ADRs). ${ }^{7}$ However, the prevalence of such harms is not well studied in children and adolescents.

Box 1. EBM facts: Information on diclofenac from the BNF for Children ${ }^{5}$ Formulations

- tablet (immediate and modified release);

- capsule;

- oral solution/suspension;

- injectable solution;

- eye drops; and

- suppository.

\section{Indications for use}

- Inhibition of intraoperative miosis during cataract surgery (eye drops);

- postoperative inflammation in cataract surgery, strabismus surgery, argon laser trabeculoplasty (eye drops);

- $\quad$ pain and inflammation in rheumatic disease including juvenile idiopathic arthritis (immediate-release oral tablet [ 6 months -17 years]);

- postoperative pain (rectal [6 months - 17 years], oral, intravenous infusion [2 - 17 years], intragluteal intramuscular injection [2 - 17 years]); and

- inflammation, mild to moderate pain (rectal [6 months - 17 years], immediate-release oral tablet [6 months - 17 years], sustained-release oral tablet, capsule [12 - 17 years]).

\section{Unlicensed use}

- Not licensed for children under 1 year of age;

- suppositories not licensed for use in children under 6 years, except for use in children over 1 year for juvenile idiopathic arthritis;

- solid dosage forms containing more than $25 \mathrm{mg}$ are not licensed for use in children; and

- injection and eye drop formulations are not licensed for use in children.

\section{Side effects}

- appetite suppression;

- diarrhoea;

- dizziness;

- gastrointestinal discomfort and disorders;

- headache;

- nausea;

- $\quad$ rash (discontinue use);

- vertigo; and

- vomiting, among others.

Contraindications with intravenous use:

- avoid injections containing benzyl alcohol in neonates;

- dehydration;

- history of asthma, confirmed or suspected cerebrovascular bleeding or haemorrhagic diathesis; 
- hypovolaemia; and

- operations with high risk of haemorrhage.

with systemic use:

- active gastro-intestinal bleeding or ulceration;

- avoid suppositories in proctitis;

- cerebrovascular disease;

- history of gastro-intestinal bleeding or perforation related to previous NSAID therapy;

- history of recurrent gastro-intestinal haemorrhage or ulceration (two or more distinct episodes);

- ischaemic heart disease;

- mild to severe heart failure; and

- peripheral arterial disease.

Since 1979, 155 people aged $\leq 19$ years have reported suspected ADRs from diclofenac to the UK's Medicines and Healthcare products Regulatory Agency (MHRA) Yellow Card Scheme, see Figure 1. Most of the ADRs (74\%, 115 of 155) were classified as serious, and seven (5\%) had a fatal outcome. ${ }^{8}$ A Cochrane systematic review published in 2015 investigated the efficacy and safety of diclofenac for acute pain in children. ${ }^{9}$ In the analysis of serious adverse events, there were 74 trials of 3611 children, of whom 26 experienced serious adverse events. Twenty-two gastrointestinal complications and two fatalities were identified from case reports. One death resulted from a gastric bleed that led to peritonitis and the other was attributed to bronchospasm from an allergic reaction. Among 500 coroners' PFD reports on medicines-related deaths between 2015 and 2016 there was one report of the death of an adolescent following missed complications from long-term use of diclofenac. ${ }^{10}$ In this BMJ EBM Coroners' Concerns to Prevent Harms article we examine this case further and explore the safety of diclofenac in children and adolescents.

Figure 1: Trends in the number of people $(n=155)$ aged $\leq 19$ years old reporting suspected adverse drug reactions (ADRs) from diclofenac to the UK's Medicines and Healthcare products Regulatory Agency (MHRA) Yellow Card Scheme. Most of the ADRs (74\%, 115 of 155) were classified as serious (green), 21\% (33 of 155) were non-serious (orange), and seven (5\%) had a fatal (blue) outcome. These data were obtained from the Yellow Card Scheme website ${ }^{11}$; pandas, ${ }^{12}$ seaborn, ${ }^{13}$ and matplotlib ${ }^{14}$ packages in Jupyter Notebooks (Python v3) were used to create the plot. The data and code are openly available at Github. ${ }^{8}$

\section{A preventable death from complications related to diclofenac}

In 2016, a 16-year-old girl with a complex medical history, including cerebral palsy, scoliosis, and visual impairment, presented to the emergency department with abdominal discomfort and distension. She was advised to present by her general practitioner (GP) who had documented his examination findings, her medical history and a medication list, and sent these to the hospital. Her medication list included diclofenac, but the indication for its use was not disclosed in the coroner's report. The medical notes and medication list were not seen by the clinical team. She was reviewed by a triage nurse and then a junior doctor, who recorded that she had a tachycardia and a soft but tender abdomen on examination. The working diagnosis was 
constipation. After discussion with the supervising Consultant, the plan was to carry out electrocardiography (ECG) and to contact the on-call Paediatrician. The ECG showed a sinus tachycardia, but no further work-up was performed. The patient was discharged home with Movicol, a laxative for the treatment of constipation. Clinical documentation was incomplete, and discussions with the paediatric team could not be corroborated; nor were their names recorded. Overnight, the patient deteriorated and died the following morning. The coroner concluded death by natural causes, contributed to by neglect. The death certificate recorded: 1a. Peritonitis; 1b. Perforated gastric ulcer; 2. Cerebral palsy.

The coroner's report identified three main concerns (see Box 2), which were addressed to the NHS Trust. ${ }^{2}$ Under regulation 28 of the Coroners' (Investigations) Regulations 2013, parties have a duty to respond to the coroner within 56 days of receiving the report, to outline actions taken or proposed, with a timeline for action, to prevent future deaths. At the time of writing this article (November 2020), no response from the NHS Trust was published on the Judiciary website, making it more than four years overdue. In the coroner's report ${ }^{2}$, reference is made to "some improvements" following a "Root Cause Analysis investigation", which was undertaken by the Trust. However, the findings of this investigation are not openly available, which could assist all NHS Trusts in preventing such deaths in the future.

\section{Box 2. Coroner's concerns ${ }^{2}$}

1. There was a missed opportunity to conduct further tests and observations to establish the cause of the tachycardia and to confirm the diagnosis of peritonitis, which if appropriate treatment was commenced, may have extended her life.

2. There were systemic failings in the documentation and transmission of information. Medical notes sent by the GP were not viewed by the clinical team at the hospital. The name of the specialist paediatrician was not recorded, and during the inquest neither the Paediatric registrar and Paediatric Consultant recalled being asked for advice on the case.

3. The medications received by the deceased, and the adverse effects of those medications, were not considered during diagnosis. Specifically, the significance of diclofenac, and the risk of peptic ulceration after prolonged use, or in high doses, was not taken into account.

\section{Safety of diclofenac in children and adolescents}

Despite diclofenac's efficacy in improving acute pain ${ }^{515}$, this death demonstrates that harms from diclofenac may go undetected or misdiagnosed in adolescents. In the analysis of MHRA's Yellow Card Scheme data, there were 415 suspected ADRs in 155 children and adolescents who had taken diclofenac. ${ }^{8}$ Most $(20 \%, 85$ of 415$)$ were gastrointestinal complications (see Figure 2); six (7\%) of these were fatal, 71 (84\%) were classified as serious, and eight (9\%) as non-serious events.

Figure 2: Numbers of fatal, serious, and non-serious adverse drug reactions $(n=415)$ related to different organ systems* for people aged $\leq 19$ years old in whom suspected adverse drug reactions (ADRs) to diclofenac were reported to the UK's Medicines and Healthcare products 
Regulatory Agency (MHRA) Yellow Card Scheme. Most of the suspected adverse reactions were related to gastrointestinal complications (20\%, 85 of 415 adverse events). These data were obtained from the Yellow Card Scheme website, ${ }^{11}$; pandas ${ }^{12}$, seaborn ${ }^{13}$, and matplotlib ${ }^{14}$ packages in Jupyter Notebooks (Python v3) were used to create the plot. The data and code are openly available at Github. ${ }^{8}$

*Blood: blood and lymphatic; Card: cardiac; Cong: congenital, familial and genetic; Ear; ear and labyrinth; Endo: endocrine, Eye: eye; Gastr: gastrointestinal; Genrl: general and administration site; Hepat: hepatobiliary; Immun: immune system disorders; Infec: infections and infestations; Injj: injury, poisoning and procedural; Inv: investigations; Metab: metabolic and nutrition; Musc: musculoskeletal and connective tissue; Neopl: neoplasms benign, malignant and unspecified; Nerv: nervous system; Psych: psychiatric; Renal: renal and urinary; Repro: reproductive system and breast; Resp: respiratory, thoracic and mediastinal; Skin: skin and subcutaneous; SocCi: social circumstances; Surg: surgical and medical procedures; Vasc: vascular.

While the death in this article demonstrates the potential for harm from diclofenac in adolescents, and the capacity for lethality if misdiagnosed or undetected, the full extent of this problem cannot be elucidated from this case report alone. Children and adolescents are more vulnerable to medication errors and adverse drug effects than adults ${ }^{16}$, and adverse events tend to occur more often in children with complex medical needs. The Royal College of Paediatrics and Child Health and the Royal College of Emergency Medicine have recommended that children with complex medical conditions should have an emergency care plan developed with families, in a similar fashion to a child's personal health record or 'red book'. ${ }^{17}$ This alleviates the burden of parents and carers having to repeat their history, and ensures that their full medical and medication history is taken into account.

Increasing knowledge, recognition, and reporting of medication harms in children and adolescents is paramount. Reporting suspected ADRs to monitoring units, such as the MHRA's Yellow Card Scheme ${ }^{11}$ and the WHO's Uppsala Monitoring Centre ${ }^{18}$, enables doctors, nurse, pharmacists, and the public to contribute to improving the safety of medicines. We therefore encourage the concerns outlined in this report to be addressed and shared across NHS trusts to educate parents and prevent future deaths in children and adolescents related to diclofenac and other NSAIDs.

\section{Declarations of interests}

ETT is financially supported by a University of Oxford Clarendon Scholarship to study for the degree of Doctor of Philosophy (DPhil/PhD). GCR is financially supported by the National Institute of Health Research (NIHR) School for Primary Care Research (SPCR), the Naji Foundation, and the Rotary Foundation to study for the degree of Doctor of Philosophy (DPhil/PhD). GCR is an Editorial Registrar of BMJ Evidence Based Medicine and is developing the https://preventabledeathstracker.net/ website. The views expressed are those of the authors and not necessarily those of the NHS, the NIHR, or the Department of Health and Social Care. 


\section{References}

1. Richards GC, Aronson JK, Heneghan C. Coroners' concerns to prevent harms: a series of coroners' case reports to serve patient safety and educate the public, clinicians and policy-makers. BMJ Evidence-Based Medicine 2020 doi: 10.1136/bmjebm-2020-111567 [published Online First: 01 Dec 2020]

2. Siddique Z. Kinga Cieciorska 2016 [Available from: https://www.judiciary.uk/publications/kinga-cieciorska/

3. EBM DataLab. Search GP prescribing data University of Oxford EBM DataLab; 2020 [Available from: https://openprescribing.net/analyse/\#org=CCG\&numlds=1001010AG, $1203040 \mathrm{AA}, 11080$ 20A0,1001010C0,1003020U0,1308010W0\&denom=nothing\&selectedTab=chart accessed November 152020.

4. Alfaro RA, DD. D. Diclofenac: StatPearls Publishing; 2020 [Available from: https://www.ncbi.nlm.nih.gov/books/NBK557879/ accessed November 182020.

5. British National Formulary for Children. Diclofenac sodium: National Institute for Health and Care Excellence; 2020 [Available from: https://bnfc.nice.org.uk/drug/diclofenacsodium.html\#medicinalForms accessed November 12020.

6. National Institute for Health and Care Excellence. Non-steroidal anti-inflammatory drugs: National Institute for Health and Care Excellence; 2015 [updated February 182018. Available from: https://www.nice.org.uk/advice/ktt13/chapter/Prescribing-data-metrics-orsupporting-resources accessed November 62020.

7. Pirmohamed M, James S, Meakin S, et al. Adverse drug reactions as cause of admission to hospital: prospective analysis of 18820 patients. BMJ 2004;329(7456):15-9. doi: 10.1136/bmj.329.7456.15

8. Richards G. Diclofenac Yellow Card Analysis: GitHub; 2020 [Available from: https://github.com/georgiarichards/diclofenac yellowcard accessed 4 Dec 2020.

9. Standing J, Savage I, Pritchard D, et al. Diclofenac for acute pain in children. Cochrane Database Syst Rev 2015;7:CD005538. doi: 10.1002/14651858.CD005538.pub3

10. Ferner RE, Easton C, Cox A. Deaths from Medicines: A Systematic Analysis of Coroners' Reports to Prevent Future Deaths. Drug Saf 2018;41:103-10. doi: 10.1007/s40264-0170588-0

11. Yellow Card. Interactive drug analysis profile: Diclofenac: Medicines and Healthcare products Regulatory Agency; 2020 [Available from: https://info.mhra.gov.uk/druganalysisprofiles/dap.html?drug=./UK EXTERNAL/NONCOMBINED/UK NON 000100012917.zi p\&agency=MHRA accessed November 92020.

12. PANDAS Development Team. pandas-dev/pandas: PANDAS. Zenodo 2020 [Available from: https://doi.org/10.5281/zenodo.3715232.

13. Waskom M. mwaskom/seaborn. Zenodo 2020 [Available from: https://doi.org/10.5281/zenodo.592845.

14. Hunter J. Matplotlib: a 2D graphics environment. Comput Sci Eng 2007;9:90-5. doi: 10.1109/MCSE.2007.55

15. Clark E, Plint AC, Correll R, et al. A randomized, controlled trial of acetaminophen, ibuprofen, and codeine for acute pain relief in children with musculoskeletal trauma.

Pediatrics 2007;119:460-7. doi: 10.1542/peds.2006-1347 
16. Stone BL, Boehme S, Mundorff MB, et al. Hospital admission medication reconciliation in medically complex children: an observational study. Arch Dis Child 2010;95:250-5. doi: 10.1136/adc.2009.167528

17. Intercollegiate Committee for Standards for Children and Young People in Emergency Care Settings. Children and young people with complex medical needs: Royal College of Paediatrics and Child Health; 2014 [Available from: https://www.rcpch.ac.uk/sites/default/files/Children and young people with complex medical needs.pdf accessed November 12020.

18. Uppsala Monitoring Centre. VigiBase. : World Health Organization Collaborating Centre for International Drug Monitoring. ; 1968 [Available from: https://www.whoumc.org/vigibase/vigibase/ accessed November 102020. 